\title{
Unravelling the complex behavior of Mrk 421 with simultaneous X-ray and VHE observations during an extreme flaring activity in April 2013
}

\section{Babic A.*}

University of Zagreb, Faculty of Electrical Engineering and Computing, Zagreb, Croatia

E-mail: ana.babicafer.hr

\section{Hassan T.}

DESY, Zeuthen, Germany

IFAE, Universitat AutÃ̌̌noma de Barcelona, Bellaterra, Spain

Paneque D.

Max Planck Institute for Physics, Munich, Germany

for the MAGIC Collaboration ${ }^{\dagger}$

\section{Balokovic M.}

Harvard-Smithsonian Center for Astrophysics, Cambridge MA, USA

Finke J.

US Naval Research Laboratory, Washington DC, USA

\section{Petropoulou M.}

Princeton University, Princeton NJ, USA

36th International Cosmic Ray Conference -ICRC2019-

July 24th - August 1st, 2019

Madison, WI, U.S.A.

\footnotetext{
* Speaker.

${ }^{\dagger}$ https://magic.mpp.mpg.de/. For collaboration list see PoS(ICRC2019)1177
} 
Mrk 421 is one of the two brightest and nearest blazars (the other one being Mrk 501). Due to their proximity and brightness, they have been the subject of a coordinated multi-instrument observational campaign since 2009, covering the spectrum from radio to VHE bands [1, 2]. Here we present a data set covering an exceptional flaring activity from April 11 to April 192013, which was collected as part of this multi-year and multi-instrument campaign. During this flare, in 42 hours of observations, MAGIC recorded the highest ever flux for this source to date ( $\sim 15 \times$ CrabFlux), and VERITAS recorded the second highest flux in 27 hours of observations [3].

Another exceptional circumstance was the excellent coverage in the hard X-ray band by NuSTAR, with 80 hours of observations. The high flux in the VHE and X-ray bands, and the 43 hours of strictly simultaneous X-ray and VHE observations, allowed us to study correlations and compare flux variability between three X-ray bands (3-7 keV, 7-30 keV and 30-80 keV) and three VHE $\gamma$-ray bands $(0.2-0.4 \mathrm{TeV}, 0.4-0.8 \mathrm{TeV}$ and $>0.8 \mathrm{TeV})$ on timescales as short as 15 minutes.

The fluxes and the variability observed in radio, optical and $\mathrm{MeV} / \mathrm{GeV}$ bands are similar to those measured in January to March 2013 [4], when the source was in a low VHE state. There is no indication of flux enhancement or variability at level similar to the ones observed in the X-ray and VHE gamma ray bands.

The fractional variability $F_{v a r}$ vs. energy shows a double-bump structure, roughly following the structure of SED. This has been observed many times for Mrk 421, and also often seen in blazars, where energy bands on falling edges of synchrotron and inverse-Compton bumps display increased variability. However, during the 9-day flaring activity from April 2013, the $F_{v a r}$ values for the highest X-ray and VHE energies are much higher than those from the January-to-March 2013 low activity reported in [4]. Even though the intra-night flares are seen in the light curves, the single-night $F_{v a r}$ values are much lower than for the 9-day activity, indicating that the processes with timescales of $\gtrsim 1$ day dominate the variability at X-ray and VHE over the processes that have timescales of hours.

The intra-night flux variations were quantified as a combination of a monotonically increasing or decreasing 'Slow' component, and an exponentially increasing and decreasing 'Fast' component. It is found that the rise and fall times are similar in all the observed instances of fast-flares. Moreover, for the flares observed simultaneously in VHE and X-rays, rise/fall times are similar in all six bands. This suggests that the mechanism generating at least some of the sub-hour flux variability is energy-independent, i.e. achromatic.

All the VHE gamma-ray bands are positively correlated with X-ray bands, when considering the full 9-day flaring activity. But the levels of correlation change substantially between different bands. We found that the strongest correlation occurs between the lowest X-ray band (3-7 keV) and the highest VHE gamma-ray band $(>0.8 \mathrm{TeV})$, with approximately linear dependence of the VHE flux on the X-ray flux, and it decreases when we modify the X-ray or VHE gamma-ray band.

We consider explaining the X-ray and VHE gamma-ray variability within a theoretical frame- 
work of a magnetic reconnection layer moving relativistically within the jet of Mrk 421 [5]. Within this model, the fast flux variations (which appear to be achromatic) would be dominated by the emission from a single plasmoid, while the multi-hour flux variations would be produced by the combined emission of many plasmoids.

The rich data set obtained during the 9-day flaring activity in April 2013 demonstrates a large degree of complexity in the variability and correlation patterns in Mrk 421, and will be further elucidated in detailed broadband SED studies. This level of complexity may be present in other blazars, but may be more difficult to observe due to the insufficient temporal temporal and energy coverage of the observations.

\section{Acknowledgements}

This research has been supported by the European Regional Development Fund under the grant KK.01.1.1.01.0009 (DATACROSS). We would like to thank the Instituto de Astrofisica de Canarias for the excellent working conditions at the Observatorio del Roque de los Muchachos in La Palma. The financial support of the German BMBF and MPG, the Italian INFN and INAF, the Swiss National Fund SNF, the ERDF under the Spanish MINECO (FPA2015-69818-P, FPA2012-36668, FPA2015-68378-P, FPA2015-69210-C6-2-R, FPA2015-69210-C6-4-R, FPA2015-69210-C6-6-R, AYA2015-71042-P, AYA2016-76012-C3-1-P, ESP2015-71662-C2-2-P, FPA2017-90566-REDC), the Indian Department of Atomic Energy, the Japanese JSPS and MEXT, the Bulgarian Ministry of Education and Science, National RI Roadmap Project DO1-153/28.08.2018 and the Academy of Finland grant nr. 320045 is gratefully acknowledged. This work was also supported by the Spanish Centro de Excelencia "Severo Ochoa" SEV-2016-0588 and SEV-2015-0548, and Unidad de Excelencia "Maria de Maeztu" MDM-2014-0369, by the Croatian Science Foundation (HrZZ) Project IP-2016-06-9782 and the University of Rijeka Project 13.12.1.3.02, by the DFG Collaborative Research Centers SFB823/C4 and SFB876/C3, the Polish National Research Centre grant UMO-2016/22/M/ST9/00382 and by the Brazilian MCTIC, CNPq and FAPERJ.

\section{References}

[1] Abdo, A. A., Ackermann, M., Ajello, M., et al., Insights into the High-energy gamma-ray Emission of Markarian 501 from Extensive Multifrequency Observations in the Fermi Era, Astrophysical Journal 727, 129 (2011)

[2] Abdo, A. A., Ackermann, M., Ajello, M., et al, Fermi Large Area Telescope Observations of Markarian 421: The Missing Piece of its Spectral Energy Distribution, Astrophysical Journal 736, 131 (2011)

[3] Benbow, W., Highlights from the VERITAS AGN observation program, 6th International Symposium on High Energy Gamma-Ray Astronomy, 1792, 050001 (2017)

[4] Baloković, M., Paneque, D., Madejski, G., et al., Multiwavelength Study of Quiescent States of Mrk 421 with Unprecedented Hard X-Ray Coverage Provided by NuSTAR in 2013, Astrophysical Journal 819, 156 (2016)

[5] Petropoulou, M., Giannios, D., \& Sironi, L., Blazar flares powered by plasmoids in relativistic reconnection, Monthly Notices of the RAS 462, 3325 (2016) 